\title{
Is theory of mind related to social dysfunction and emotional problems in $22 q 11.2$ deletion syndrome (velo-cardio-facial syndrome)?
}

\author{
Linda E. Campbell • Angela F. Stevens • Kathryn McCabe • Lynne Cruickshank • \\ Robin G. Morris • Declan G. M. Murphy • Kieran C. Murphy
}

Received: 10 October 2010 / Accepted: 5 April 2011 /Published online: 5 May 2011

(C) Springer Science+Business Media, LLC 2011

\begin{abstract}
Social dysfunction is intrinsically involved in severe psychiatric disorders such as depression and psychosis and linked with poor theory of mind. Children with 22q11.2 deletion syndrome (22q11DS, or velo-cardio-facial syndrome) have poor social competence and are also at a particularly high risk of developing $\operatorname{mood}(40 \%)$ and psychotic (up to $30 \%$ ) disorders in adolescence and young adulthood. However, it is unknown if these problems are associated with theory of mind skills, including underlying social-cognitive and social-perceptual mechanisms. The present cross-sectional study included classic social-
\end{abstract}

L. E. Campbell • A. F. Stevens · R. G. Morris · D. G. M. Murphy Institute of Psychiatry, King's College London,

University of London,

London, UK

\section{E. Campbell ( $\square)$}

School of Psychology,

University of Newcastle,

Science Offices Ourimbah, NSW 2258, Australia

e-mail: Linda.E.Campbell@newcastle.edu.au

\section{Cruickshank}

School of Psychology,

University of Newcastle,

PO Box 833, Newcastle, NSW 2300, Australia

\section{K. McCabe}

Centre for Brain and Mental Health Research,

University of Newcastle,

PO Box 833, Newcastle, NSW 2300, Australia

\section{K. C. Murphy}

Department of Psychiatry, Royal College of Surgeons in Ireland,

Dublin, Ireland

\section{K. McCabe}

Schizophrenia Research Institute,

Sydney, Australia cognitive false-belief and mentalising tasks and socialperceptual face processing tasks. The performance of 50 children with 22q11DS was compared with 31 age-matched typically developing sibling controls. Key findings indicated that, while younger children with 22q11DS showed impaired acquisition of social-cognitive skills, older children with 22q11DS were not significantly impaired compared with sibling controls. However, children with 22q11DS were found to have social-perceptual deficits, as demonstrated by difficulties in matching faces on the basis of identity, emotion, facial speech and gaze compared with sibling controls. Furthermore, performance on the tasks was associated with age, language ability and parentally rated social competence and emotional problems. These results are discussed in relation to the importance of a better delineation of social competence in this population.

Keywords 22q11.2 deletion syndrome - Velo-cardio-facial syndrome $\cdot$ Social functioning . Theory of mind $\cdot$ Socialperception $\cdot$ Social-cognition

\section{Background}

Social competence is a multidimensional construct in which social, emotional, cognitive and behavioural skills are involved in a dynamic interplay with the environment. This, in turn, facilitates successful social adaptation, including the ability to initiate and maintain satisfactory relationships, with, for example, peers (Iarocci et al. 2007). One way to explore the complex genetic and environmental interactions modulating social competence is to study individuals with a known genetic disorder who also have differences in social behaviour. The 22q11.2 deletion syndrome (22q11DS) also known as velo-cardio-facial 
syndrome is the most common known microdeletion disorder and occurs in one in every 2,000 to 4,000 live births (Shprintzen 2005; Vorstman et al. 2006). The syndrome has a large phenotypic spectrum but is most commonly associated with developmental anomalies such as cardiac and palatal abnormalities, a syndrome specific typical face, intellectual disabilities and specific social and cognitive impairments. Children with 22q11DS are frequently described as being shy and withdrawn, socially immature and as having difficulties with initiating and maintaining positive peer relationships (Golding-Kushner et al. 1985; Heinemande Boer et al. 1999; Swillen et al. 1997, 1999; Shprintzen 2000). Children with the syndrome also present with a high rate of psychiatric disorders including autism spectrum disorder, attention-deficit disorder, separation anxiety and affective disorders (Vorstman et al. 2006; Fine et al. 2005; Gothelf et al. 2004; Swillen et al. 2000). The syndrome is further believed to be the third highest known risk factor for developing schizophrenia-like psychotic disorders in late adolescence or early adulthood (Murphy 2002). Many of the psychiatric disorders experienced by people with 22q11DS are associated with a lack of appropriate social competence, and it has been suggested that individual differences in social competence among people with 22q11DS may be associated with the subsequent development of psychiatric disorders such as anxiety and depression (Murphy 2005). Research over the last decade has shown that social functioning in psychiatric disorders such as depression and psychosis (Wang et al. 2008) are linked with deficits in theory of mind, for instance, the ability to judge one's own and other people's mental states (Premack and Woodruff 1978).

The ability to accurately understand, reason and predict other people's behaviour requires the integration of complex skills. It has been argued that theory of mind skills are dependent on two dissociable components, namely a socialperceptual and a social-cognitive component (Tager-Flusberg and Sullivan 2000). The social-perceptual component includes the ability to recognise people and to interpret people's mental state from facial emotions or body expressions. In healthy individuals, the processing of human faces and, in particular, the ability to accurately recognise facial emotions is vital for social competence and is thought to depend on specialised neural systems including the occipitotemporal cortex (for a review, see (Posamentier and Abdi 2003)). More specifically, it has been suggested that while invariant aspects of faces are dependent on the lateral fusiform gyrus, more changeable aspects such as expression, eye gaze and lip movement are processed in the superior temporal sulcus and associated networks (Haxby et al. 2000). Few studies have specifically examined face processing in people with 22q11DS; however, recently, two functional magnetic resonance imaging (fMRI) studies identified atypical neural activations among children and adults with
22q11DS compared with healthy (Andersson et al. 2008) and learning-disabled comparison subjects (van Amelsvoort et al. 2006) when processing facial expressions. The differences in neural activations were argued to be face-specific and not due to a general visual perceptive deficit since the neural activation pattern in people with 22q11DS were similar to controls when presented with non-face stimuli such as houses (Andersson et al. 2008). Likewise, it appears as if young adults with 22q11DS use atypical strategies while viewing photographs of faces displaying emotions (as measured using visual scanpath technology), and this is associated with poorer accuracy when labelling the displayed emotions (Campbell et al. 2010a). In particular, it was reported that people with 22q11DS spent more time looking at peripheral (off-the-face) rather than internal (eye, nose, mouth) features of the face. The young people with 22q11DS also spent less time looking at the eye region of the face and significantly more time looking at the mouth compared with controls. These studies indicate that people with 22q11DS do not process faces in a typical manner; it also appears as if these atypical processes are associated with poorer skills of encoding and interpreting facial information. Indeed, it has been suggested that short-term memory of unknown faces may be impaired in 22q11DS. In particular, when children with 22q11DS were asked to recognise faces that had been learned immediately before, they performed poorer not only compared with controls but also compared with their performance on other memory tasks (e.g., visual-spatial; Campbell 2006; Lajiness-O’Neill et al. 2005). Furthermore, we recently reported that children with 22q11DS (a subgroup of those reported in the current paper) had a significantly reduced performance on face processing tests of gaze direction, identity and emotion recognition compared with intellectually, age- and gender-matched children with Williams syndrome (WS; Campbell et al. 2009). However, no significant group differences were identified on a facial speech-recognition task (Campbell et al. 2009). These findings have led to the assumption that face processing is atypical in people with 22q11DS. However, since people with WS usually perform in the normal range and significantly better than mental-agematched controls on such tasks (Tager-Flusberg et al. 2003), we still need to evaluate the face processing skills of people with 22q11DS compared with typically developing controls in order to determine how people with 22q11DS process faces.

A second key component of theory of mind is described by Tager-Flusberg and Sullivan (2000) as social-cognitive. The social-cognitive component underlies the ability to understand that other people have mental states that are independent from one's own including independent thoughts, beliefs and intentions and to make attributions about these (Castelli et al. 2002). Social-cognitive skills are crucial to understand and correctly predict peoples' actions and are often measured using classical false-belief tasks such as the Sally-Ann 
scenario (Baron-Cohen et al. 1985) and mentalising tasks such as the Strange Stories (Happé 1994; Jolliffe and BaronCohen 1999). The neurobiological substrate most strongly linked with the social-cognitive component is the medial frontal region of the brain (Siegal and Varley 2002). Recently, it was reported that a sample of children (Niklasson et al. 2002) and adults (Bassett et al. 2007; Chow et al. 2006) with 22q11DS had theory of mind deficits. These studies were valuable first steps. However, one study (Niklasson et al. 2002) did not include a control group, and the other study focussed solely on adults with 22q11DS and schizophrenia (Bassett et al. 2007; Chow et al. 2006). In contrast, we recently reported that children with 22q11DS, compared with matched children with WS, did not perform poorer on false-belief tasks (Campbell et al. 2009). There were some group differences (with the 22q11DS group performing poorer) on the Strange Stories task, although the result may have been confounded by low comprehension or on stories requiring mentalising skills (Campbell et al. 2009). However, the study was limited by a small sample size and did not examine how children with 22q11DS performed compared with typically developing children.

The objectives of the current study were to examine the two proposed components of theory of mind, socialperception (face processing) and social-cognitive (false beliefs) in a cohort of children with 22q11DS compared with typically developing sibling controls. Furthermore, we aimed to investigate if performance on these tasks was related to everyday social competence and emotional problems, as rated by the parent(s). We tested the hypotheses that: compared with age- and gender-matched sibling controls (1) children with 22q11DS participants have specific deficits in face processing tasks most related to social competence, i.e., emotion recognition and gaze direction; (2) children with 22q11DS show a deficit on the false-belief and mentalising tasks; and (3) that parent-rated social competence is correlated with performance on these theory of mind tests (face processing tasks, false-belief and mentalising tasks)

\section{Methods}

\section{Participants}

There were 50 participants in the 22q11DS group (22 males, 28 females; age range, 6 to 16.75 years $(\mathrm{M}=10.99, \mathrm{SD}=$ $2.90)$ ). The presence of a $22 \mathrm{q} 11.2$ deletion was confirmed through the use of fluorescence in situ hybridisation. Thirtyone unaffected sibling controls, matched for age (18 males, 13 females; age range, 6 to 14.75 years $(\mathrm{M}=10.62, \mathrm{SD}=2.59))$ were also included in the study. The majority of participants were of white Caucasian descent (79 out of 81). None of the participants in this study presented with the clinical phenotype of 22qDS but without the large $3 \mathrm{Mb} 22 \mathrm{q} 11.2$ deletion. As such, all participants were included in analysis. Furthermore, those with a clinically detectable medical disorder known to affect brain structure (e.g. epilepsy or hypertension) and a history of head injury or stroke were excluded. We recruited children with 22q11DS and their typically developing siblings through the VCFS-UK support group. We chose to compare the 22q11DS cohort with sibling controls at a group-level for several reasons. First, 22q11DS is a random de novo gene deletion, as such attenuated forms of the condition are not present in siblings. Second, the sample was selected in order to control for socio-economic status as well as home environment and to facilitate recruitment.

According to independent sample $t$ tests, there was no significant difference in age $(t=0.59, \mathrm{df}=79, p=0.56)$ or gender $\left(\chi^{2}=1.23, \mathrm{df}=79, p=0.22\right)$ between the 22q11DS and the control group (see Table 1). Prior to commencing the study, the participant's parents/guardians and, in cases where the participant was 16 years or older, the participant, gave written informed consent after the procedure was fully explained. A subgroup of the current participants has been included in a study of brain structure $(N=39$; Campbell et al. 2006) and in a comparison of children with 22q11DS to children with Williams syndrome $(N=15$; Campbell et al. 2009). The study was approved by the local ethics committee at the Institute of Psychiatry, King's College, London, UK.

\section{Materials}

Intellectual function was measured using the Wechsler Intelligence Scale for Children version III UK edition (Wechsler 1991), an intelligence test for children aged 6 to 16 years old, consisting of 13 subtests which can be used to generate the participants Full-Scale IQ, Performance IQ and Verbal IQ. Furthermore, to investigate the influence of language ability on the experimental task, the British Picture Vocabulary Scale (BPVS; Dunn, Whetton, and Burley, 1982) and the Test for Reception of Grammar (TROG; Bishop 1983) were included. The BPVS is a test of receptive (hearing) language, in which the individual matches a word presented orally with one out of four pictures by pointing. The TROG measures understanding of grammatical contrasts. The tests are designed to remove cues (such as contextual) to aid the understanding of the sentence, leaving the child with only the grammatical structure to aid them in interpreting the sentence accurately. Each sentence presented to the child has four options presented pictorially, and the items contain both grammatical and non-grammatical (lexical) distractors in order to determine whether the child has a specific problem with grammatical understanding or a bad performance due to other factors such as poor attention or memory. 
Table 1 Demographic and behavioural data

\begin{tabular}{llccc}
\hline & Group & Mean & SD & Range \\
\hline Chronological age & 22q11DS & 11.00 & 2.9 & $6-16$ \\
Full-scale IQ & Controls & 10.94 & 2.62 & $6-15$ \\
& 22q11DS & 65.80 & 9.32 & $40-94$ \\
Digit span & Controls & 104.16 & 12.91 & $72-133$ \\
& 22q11DS & 6.23 & 2.09 & $1-11$ \\
TROG & Controls & 9.63 & 2.83 & $4-16$ \\
& 22q11DS & 13.34 & 3.99 & $3-19$ \\
BPVS & Controls & 17.23 & 2.46 & $8-20$ \\
\multirow{2}{*}{ SDQ peer relationship problems } & 22q11DS & 79.92 & 14.10 & $43-108$ \\
& Controls & 104.67 & 11.79 & $86-127$ \\
SDQ emotional problems & 22q11DS & 3.74 & 2.57 & $0-10$ \\
& Controls & 1.04 & 1.88 & $0-7$ \\
& 22q11DS & 4.31 & 3.19 & $0-10$ \\
& Controls & 1.38 & 1.74 & $0-6$ \\
\hline
\end{tabular}

\section{Face processing}

This was measured using the MRC Face Processing Skills Battery, a procedure that has previously been shown to be an effective tool for research with children with developmental disorders (Bruce et al. 2000). It consists of 14 tests which examine four different aspects of face processing; Identity, Emotion, Eye gaze and Facial Speech (Sound). In the current study, each test included images of children's faces (unless otherwise stated) on a uniform grey background approximately $5.5 \times 4 \mathrm{~cm}$ in size printed on A4 paper. The tests require pointing responses and increases in difficulty across trials.

There are five Identity tests with 16 trials each, in which the participant is required to indicate which face out of two choices belong to the same child as another face presented above them. For Idmatch.dis, the two options are dissimilar in appearance in terms of age, gender or general appearance whilst, for Idmatch.sim, the two options are similar in this regard; Idno.dis has the same faces as Idmatch.dis, but the hair and ears are removed; Idno.sim task has the same faces as Idmatch.sim but the hair and ears are removed; finally, Idmask has the same faces as Idno.sim but with grey circles are painted over the eyes.

There are three Emotion tests consisting of 12 trials each, in which images of happy, sad, angry or surprised facial expressions are presented in equal proportions. Expair is used to determine whether the participant can identify an emotional expression given the verbal label. Pairs of faces are shown, and the participant indicates which face is 'happy', 'sad', 'angry' or 'surprised.' For Exmatch.child and Exmatch.adult, the participant indicate which of two presented faces 'feel the same way' as an above presented facial image. Exmatch.adult differs from Exmatch.child in that it uses adult's faces.
There are three tests of Gaze directionality. In Gazepair, two faces are presented and the task is to decide which face is looking at the participant with the position of the head being either full face or 3/4 view. Gazematch.45 and Gazematch.10 requires the participant to indicate which of two faces are looking in the same direction as an above face. Gazepair and Gazematch.45 uses 12 trials with children's faces while Gazematch.10 uses trials with one adult male's face.

There are three Facial Speech tests. Facial speech refers to the lip movements associated with the expression of basic speech sounds required for verbal communication (e.g. "ee", "oo", etc.). In this task, the mouths on the images were saying "aa", "ee", "ff" or "oo" in equal proportions. In Soupair, 12 pairs of faces are presented, and the participant is required to indicate which is saying either "aa", "ee", "ff" or "oo". The Soumatch.ff and Soumatch.44 tests require the participant to indicate which of two faces is making the same sound as an above image. Soumatch.ff have 12 trials with the faces shown in full face views while for Soumatch. 44 have 24 trials in which the top face shown is a 3/4 view and the bottom faces are shown in full face view.

The Idmatch.sim, Expair, Exmatch.child, Gazepair, Gazematch.45, Soupair and Soumatch.ff tests were administered to the participant first. Participants were required to score greater than $80 \%$ accuracy for tasks in this grouping. If they made this cutoff, they progressed to the second level of face processing tasks. The second level of tasks are considered more complex, and administration continued until the participant was no longer able to maintain the predefined $(80 \%)$ level of accuracy.

\section{False-belief and mentalising}

The tasks were selected on the basis that they have good validity and fair to moderate reliability for children with 
varying intellectual abilities including those with developmental disorders (Hughes et al. 2000).

The Sally-Anne is a first-order false-belief task in that it examines the ability to understand that others can have beliefs that differ from their own and requires them to predict another person's behaviour accordingly (BaronCohen et al. 1985). Two dolls named "Sally" and "Anne" are shown to act out a false-belief scenario in which a marble is displaced whilst Sally is not looking. In order to pass this task, the participant has to correctly respond to the false-belief question "Where will Sally look for her marble?" The participant is also asked "Where is the marble really?" (reality question) and "Where was the marble in the beginning?" (memory question). The child could score a pass or fail on the false-belief question.

In the Smarties task, another first-order test, the participants themselves experiences having a false-belief (Gopnik and Astington 1988). Specifically, the participant is shown a Smarties box (which usually contains chocolates) and is asked what they think is in the box. The experimenter then opens the box and shows the participant that there is a pencil inside. The box is then re-closed with the pencil inside, and the participant is asked what they think their parent (who has not seen what is inside the box) would say is inside the box. The participant pass the task if they respond that their parent will say there are Smarties in the box but fails if they respond that the parent will think the box contained a pencil.

The Chocolate task is a second-order false-belief task, which examines the ability to think about what a person falsely believes another person believes (Perner and Wimmer 1985). This was attempted only if both the Sally-Anne and Smarties tasks had been passed. It involves reading the participant a false-belief story concerning two fictitious children named "Mary" and "John"; the task is supplemented by pictures also portraying the story. It is similar to the smarties displacement scenario except that in this case the question refers to a second-order false-belief by asking "Where does John think Mary will look...?". The task is scored as a pass or fail.

The Strange Stories task (Happé 1994; Jolliffe and Baron-Cohen 1999) was used to assess mentalising abilities, when listening to stories by examining the ability to interpret non-literal statements. Four stories involve everyday situations where the characters say things they literally do not mean (i.e. lies, false beliefs, double bluff or manipulation). These are contrasted with four physical stories which act as a control against comprehension deficits. They differ from the mentalising stories in that they do not involve mental states and are not social in nature, involving situations in which there is an unforeseen outcome with a mechanical-physical cause. The stories are presented in an alternating manner. To minimise memory requirements printed forms of the stories are placed in front to the participant during reading and decision making. For mentalising stories, the questions are "Why did X say that?" and "Was it true what X said?" For each physical story, the participants are asked why something happened or why a particular action had taken place. For each story, two points are awarded for an accurate full description of the story, one point for a partial description, or no points if the participant refers to irrelevant information. The scores on each story type are then combined to produce single mentalising and physical stories scores for each participant.

\section{Social competence and emotional well-being}

The Strengths and Difficulties Questionnaire (SDQ; Goodman et al. 2000) was completed by a parent. The SDQ is a brief behavioural screening questionnaire for 3- to 16-year-olds, consisting of 25 items which form five clusters, Emotional Symptoms, Conduct Problems, Hyperactivity/Inattention, Peer Relationship Problems and Prosocial Behaviour. The occurrence of particular attributes are rated using a three-point Likert scale ("not true", "somewhat true" or "certainly true"). The reliability and validity of the SDQ make it a useful measure of adjustment and psychopathology in children and adolescents (Goodman et al. 2000). For the purpose of the current study, only the emotional problems and peer relationship problem scores were analysed.

\section{Data analysis}

Statistical analyses were conducted using the Software Package for the Social Sciences (SPSS) version 14. Independent sample $t$ tests were used to compare the groups on the variables SDQ peer and emotional problems. For the face processing tasks, a total score for each aspect was computed by summing the scores for each participant across the subtests, and when a participant did not progress to a higher level, a score of 0 was given for that particular subtest. For the Strange Stories and the face processing tasks, group differences were examined separately by simple linear regression analyses. The effect of age on the participants' performance were examined using an interaction term based on the product of standardised age scores $\times$ group score (22q11DS $=1$; controls $=-1$ ) enabling both group membership and age to be examined within the one variable. The beta coefficients are reported in the result section. A paired-sample $t$ test was used to examine within-group differences on the Strange Stories task. For the Sally-Anne task, Smarties task and Chocolate task, between-group comparisons were conducted using Fisher's exact tests due to small participant numbers in each category. Consistent with previous findings (Campbell et al. 2010b), IQ score (reported WASI scores; 
Wechsler 1991) was omitted from analysis as a covariate in the repeated-measures design as reported IQ differences were greater than $2 \mathrm{SD}$ between the groups and was therefore considered a group defining characteristic.

Finally, an aggregate standardised score for each of the two theory of mind components (social-perceptual $=$ face processing, social-cognitive $=$ false belief and mentalising) was computed. This was used to examine within-group correlations (using Pearson's $r$ correlations) with the measures of social competence (as measured by the mean standardised score for peer problems) as well as chronological age, full-scale IQ and standardised scores from the BPVS, the WISC-III subtest of digit span (to measure working memory) and the TROG.

\section{Results}

The 22q11DS group had significantly poorer social competence (SDQ; peer relationship problems, $p<0.0005$ ) and more emotional problems $(t=4.88, \mathrm{df}=65.26, p<$ $0.0005)$ compared with sibling controls (see Table 1).

\section{Face processing}

The data are shown in Table 2 and Fig. 1. Simple linear regression analyses revealed a significant effect of Group on the face processing tasks; Identity (beta coefficient $=-0.725$, $p<0.0005$ ), Emotion (beta coefficient $=-0.498, p<0.0005$ ), Gaze (beta coefficient $=-0.586, p<0.0005$ ), Facial speech (beta coefficient $=-0.360, p<0.0005$ ) with the sibling control group performing significantly better than the 22q11DS group. There was also a significant effect of age on Identity (beta coefficient $=0.235, p<0.005$ ), Gaze (beta coefficient $=0.226$, $p<0.02$ ) and Facial speech (beta coefficient $=0.242, p<0.03$ ), with the older participants performing better than younger participants, but no age $\times$ group interactions were identified.

Further repeated-measures analyses were conducted with Group as the between-subjects factor and each of the face processing tasks entered as the within-subjects factor. Analysis revealed a significant group $\times$ task interaction $(\mathrm{F}(1,78)=4.68, p<0.03)$. Post hoc within-group paired-sample $t$ tests found that both the 22q11DS and the control group had significantly more difficulties with the Gaze task compared with the other tasks $(p<0.02)$. Meanwhile, the control group had significantly higher scores on the Identity task compared with the other tasks $(p<0.0005)$ whilst no such pattern was identified in the 22q11DS group $(p>0.05)$.

With the exception of Soumatch.ff (4\%), all control participants advanced to the second level after achieving greater than $80 \%$ accuracy on the seven tasks used in the first level of testing. In the 22q11DS group, the proportions of participants who failed to complete the first level of testing were 10\% Idmatch.sim, 2\% Expair, 16\% Exmatch. child, 26\% Gazepair, 42\% Gazematch.45, 4\% Soupair and $14 \%$ Soumatch.ff.

\section{False-belief and mentalising}

The entire control group passed the Sally-Anne Task compared with $90 \%$ of 22q11DS group $(n=45)$, although this difference was not statistically significant (Fisher's exact test, $p=0.15$ ). All five 22q11DS participants that failed the Sally-Anne task were in the 6-9-years age group. A higher percentage of the control participants (pass= $100 \%, n=31$ ) passed the Smarties Task compared with the 22q11DS participants (pass $=95.8 \%, n=46$ ). However, there was no significant between-group difference in accuracy on the Smarties task (Fisher's exact test, $p=0.52$ ).
Table 2 Means, percent accuracy and standard deviations for performance on the Strange Stories task and the Face Processing battery across the 22q11DS and sibling control group

\begin{tabular}{|c|c|c|c|c|}
\hline & Group & Mean accuracy & $\%$ Accuracy & SD \\
\hline \multicolumn{5}{|l|}{ Strange stories } \\
\hline \multirow[t]{2}{*}{ Physical stories } & $22 q 11 D S$ & 3.92 & 49.00 & 2.25 \\
\hline & Controls & 6.10 & 76.25 & 1.52 \\
\hline \multirow[t]{2}{*}{ Mentalising stories } & 22q11DS & 2.73 & 34.12 & 2.03 \\
\hline & Controls & 6.60 & 82.5 & 1.63 \\
\hline \multicolumn{5}{|l|}{ Face processing } \\
\hline \multirow[t]{2}{*}{ Identity } & 22q11DS & 39.71 & 49.64 & 8.43 \\
\hline & Controls & 62.06 & 77.58 & 14.53 \\
\hline \multirow[t]{2}{*}{ Facial speech } & 22q11DS & 25.18 & 52.47 & 10.21 \\
\hline & Controls & 31.55 & 65.73 & 6.21 \\
\hline \multirow[t]{2}{*}{ Gaze } & 22q11DS & 12.31 & 33.46 & 5.09 \\
\hline & Controls & 18.52 & 57.86 & 1.96 \\
\hline \multirow[t]{2}{*}{ Emotion } & 22q11DS & 16.57 & 46.03 & 6.57 \\
\hline & Controls & 22.42 & 62.28 & 3.68 \\
\hline
\end{tabular}




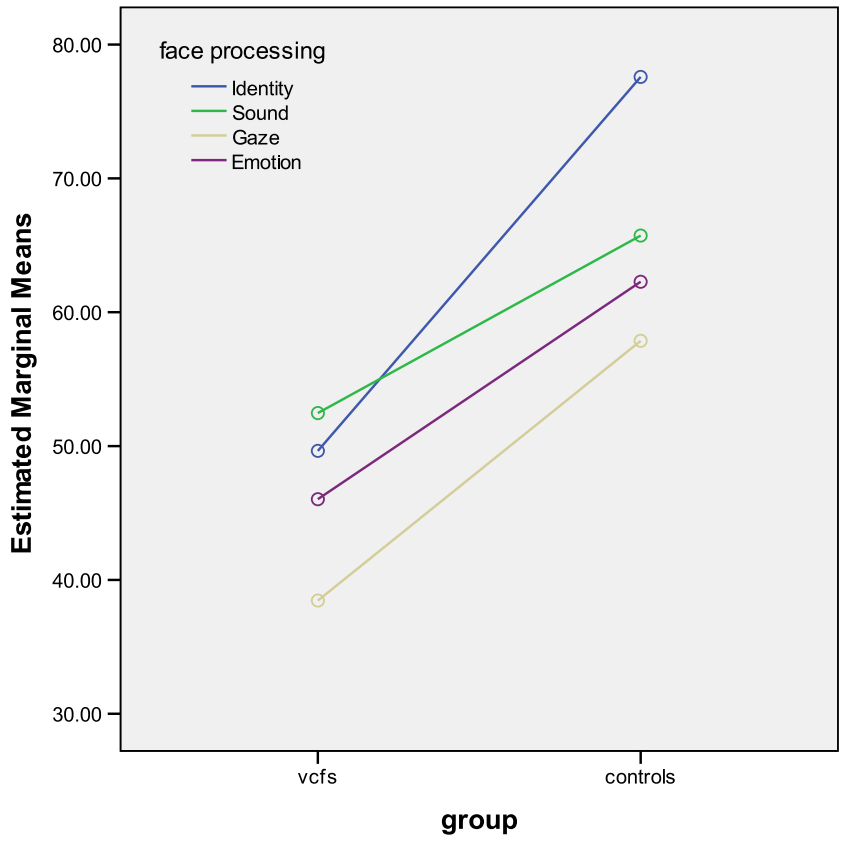

Fig. 1 Task performance on face processing battery

As mentioned previously, only participants who passed both the Sally-Anne and the Smarties task participated in the Chocolate Task (100\% of the control group and $90 \%$ of the 22q11DS group). Significantly more control participants $(100 \%, n=31)$ passed the Chocolate Task compared with the 22q11DS participants $(82.2 \%, n=37)$ (Fisher's exact test, $p=0.02$ ). The majority of participants that failed the task was between 6 to 9 years of age ( $n=7$ out of 8 ).

The 22q11DS group scored lower compared with controls on both the Strange Stories Task mentalising and physical stories (see Table 2). However, linear regression analyses on the difference scores revealed a significant effect of Group on the type of Strange Stories (beta coefficient $=0.437, p<0.0005$ ), with the 22q11DS group performing better on the physical stories compared with the mentalising stories whilst the control group performed at a comparable level in both tasks, this effect was not affected by age $(p=0.02)$.

Furthermore, to explore the potentially confounding influences of using siblings as control group, we recalculated the analyses using a repeated-measures approach linking 22q11DS participants with their related sibling control. Overall, the pattern of significant and nonsignificant findings did not differ under this approach, with the exception of one face processing task (facial speech) which was not significantly different across 22q11DS and siblings when using this type of analysis.

In the 22q11DS group, Pearson correlations showed that performance on the social-cognitive construct was significantly correlated with age $(r(45)=0.61, p<0.0005)$, and both the social-cognitive and social-perceptual constructs were correlated with accuracy on the TROG (Face processing, $r(45)=0.646, p<0.0005$; social-cognitive, $r(45)=0.347$, $p<0.015)$. In addition, the social-perceptual construct was related to working memory ability as measured by performance on the digit span $(r(45)=0.44, p<0.001)$. In the sibling control group performance on the two constructs were similarly correlated with performance on the TROG (social-perceptual $(r(30)=0.54, p<0.002)$; social-cognitive $(r(30)=0.43, p<0.02)$. However, chronological age was correlated with the social-perceptual construct $(r(30)=0.41$, $p<0.02)$ while full-scale IQ $(r(30)=0.56, p<0.001)$ and the BPVS score $(r(30)=0.37, p<0.04)$ was correlated with the social-cognitive construct.

Correlational analyses revealed that performance of both the social-perceptual $(r(49)=0.33, p<0.03)$ and the socialcognitive $(r(49)=0.3, \mathrm{p}<0.03)$ construct were related to social competence in the children with 22q11DS. In addition, the social-perceptual and social-cognitive constructs were correlated in both groups (22q11DS, $r(45)=0.32, p<0.03$; controls $r(30)=0.38, p<0.04)$. Meanwhile, only the socialperceptual construct was related to social competence in the sibling control group $(r(30)=0.38, p<0.04)$. Finally, social competence was strongly correlated with emotional problems $(r(42)=0.44, p<0.004)$ in the 22q11DS group but not in the sibling control group $(p=0.13)$.

\section{Discussion}

The current investigation is the first to investigate socialcognitive and social-perceptual mechanisms and their relationship to social competence and emotional problems among children with 22q11DS and typically developing sibling controls.

We predicted that the 22q11DS participants would have specific deficits in the social-perceptual tasks of face processing. In particular, we predicted that children with 22q11DS would have problems with tasks of a high salience to social functioning, such as emotion and gaze identification. We did indeed identify deficits in these aspects of face processing compared with sibling controls, although we also identified deficits in identity recognition and facial speech indicating a general deficit in facial processing. The greatest deficit, however, was identified in the Gaze direction task, and while this may have been at least partly due to an increased level of difficulty, we do not believe that this fully explains this finding. An independent study of visual scan path strategies in young people with 22q11DS indicate that people with the syndrome spend less time looking at the eyes compared with the mouth when judging facial emotions (Campbell et al. 2010a). This may be indicative of inefficient facial perceptual strategies 
which might have influenced the performance on the gaze identification tasks carried out in the current study. Our findings are in agreement with current neurobiological knowledge of specific brain anomalies among people with 22q11DS. In particular, it has been reported that socialperceptual ability is dependent on the occipito-temporal cortex including the lateral fusiform gyrus and also the superior temporal sulcus and associated networks (Haxby et al. 2000). It has been well established that the occipital and temporal regions of the brain are affected by a deletion at chromosome 22q11.2 (Campbell 2006; Henry et al. 2002). Findings from magnetic resonance imaging (MRI) studies support the idea that social-perceptual impairments in the 22q11DS group may be due to atypical neural structures and brain functioning and have identified less insular and frontal cortical activation and relatively more activation in bilateral occipital cortex when viewing emotional faces (Andersson et al. 2008; van Amelsvoort et al. 2006). In addition, atypical scanning patterns of photographs of emotional human faces have been revealed (Campbell et al. 2010a). However, it is still unknown what strategies children with 2211DS use to judge the identity of a person or when determining eye gaze direction or facial speech.

It is also unknown whether the observed problems in facial perception are due to a general visual perceptual impairment or a face-specific social-perceptual impairment. Although not directly comparable, we have revealed impairments in some aspects of object perception such as identifying objects from unusual viewpoints in children (Campbell 2006) and adults (Henry et al. 2002) with 22q11DS, indicating that there may be generalised problems with visual perception present in this population. However, the fMRI data reported by Andersson and colleagues did not find any group differences in neural activation when the groups were presented with objects such as houses which the authors interpreted as evidence for a face-specific neural anomaly (Andersson et al. 2008). It is also unknown if the origins of the observed face socialperceptual impairments are due to early face processing problems which may have resulted in worse social competence and hence less social interactions (and less practice) or if problems in another related area could have resulted in the observed problems. Unfortunately, no study of infant social-perception/cognition has yet been undertaken in 22q11DS, so the developmental trajectory of these skills is not clear. One could also argue that the group differences are simply due to the lower intellectual functioning of the clinical group. However, we have previously compared a subgroup of the current sample with a group of age-, gender- and intellectually matched children with Williams syndrome and subsequently identified a specific impairment in the 22q11DS group when performing the identity, emotion and gaze tasks while no significant group differences were identified in the facial speech task or the object perceptual tasks. Hence, we do not believe that the failure on these tasks are simply due to lower intellectual functioning in these children but rather a combination of lower intellectual functioning and syndrome specific differences.

However, it does seem as if language skills (in particular grammatical skills) and working memory ability are important to take into consideration when evaluating social-perceptual skills in this group of people. In the present context, consideration of semantic memory skills may elucidate the association reported between measures of face processing and grammatical skills (measured by TROG), for one who is proficient at storing and retrieving verbal concepts is liable to be proficient on tasks of verbal ability, as both require efficient access to words/concepts. Whilst the processes involved in identifying an individual are considered distinct from those required to perceive emotion and speech related actions of the mouth (Bruce and Young 1986), these processes have in common a reliance on semantic memory. As such, despite our best efforts to minimise the influence of both language and working memory in the design of the face processing tasks by using simple forced-choice matching tasks without requiring verbal responses, the findings from this study would suggest that face processing, by nature, requires these abilities.

For future studies, it will be important to compare socialperceptual skills of people with 22q11DS with people with other developmental disorders characterised by lower intellectual functioning and also to investigate if the observed facial processing deficits are specific or if similar deficits exist in other visual perceptual tasks. In addition, it would be valuable to use more naturalistic stimuli and tasks in order to determine the exact nature of the face processing deficits in 22q11DS. To conclude, considering the importance of social-perception in communication and social competence, the face processing deficits observed in the 22q11DS group needs further investigation.

We also predicted that the 22q11DS group would perform poorer on the social-cognitive tasks compared with the sibling control group. Our data suggests that socialcognitive deficits only occurred among the younger 22q11DS participants and only for more advanced second-order false-belief tasks and Strange Stories signifying that the acquisition of more complex social-cognitive tasks of false belief is suppressed in $22 \mathrm{q} 11.2$, but that this reflects a delay rather than a deficit. The influence of age on social-cognitive skills in children with 22q11DS may be related to a delay in the maturation of the frontal cortex among children with 22q11DS (Jablensky 2000). Van Amelsvoort and colleagues suggested that volumetric differences in the frontal lobes normalises somewhat in 
adults with 22q11DS Van Amelsvoort et al. 2001. Other factors such as gender and COMT phenotype has also been found to moderate frontal lobe morphology in 22q11DS and could potentially have an effect on our findings (Sands and Harrow 1995). However, our study did not identify any significant gender differences in the 22q11DS group. Future prospective studies will be designed to test this hypothesis further. In particular, it is important to include tasks that are largely independent from language to exclude the possibility that task performance is not simply attributable to language impairments. The tasks included in the current study included detailed narratives and both these, and the test-questions were grammatically complex (with the exception of the Smarties task). Indeed, our data indicate that performance on the false-belief tasks was related to grammatical competence as measured by the TROG. In addition, the pass or fail nature of the first- and secondorder false-belief tasks produced ceiling effects amongst the older participants and the sibling controls, limiting our ability to detect the range of false-belief skills present in the two groups and possibly concealing any significant between-group differences. In order to take this into account, the Strange Stories were included in the study; however, due to the complex narratives, the performance of the participants with 22q11DS may not truly reflect their mentalising ability. It will also be important to control for other cognitive processes such as inhibition and working memory.

Finally, our data suggest that social competence in the 22q11DS group is strongly associated with emotional problems, reflecting anxious and depressive traits. It has been reported that poor premorbid social functioning is related to worse outcomes among people with both depression and psychotic disorders (Jablensky 2000; Sands and Harrow 1995) and associated with poor theory of mind skills (Wang et al. 2008). Hence, children with 22q11DS with poor social competence due to underlying problems with, e.g. the social-perceptual components of theory of mind may be at particularly high risk of later psychopathology. This highlights the need to properly assess the mechanisms underlying social competence among children with 22q11DS in order to be able to design evidence-based interventions aimed at increasing resilience in this group of children at high risk of developing mood and psychotic disorders. Taken together, lack of social competence and associated emotional problems are likely to have a very significant negative impact on the quality of life and longterm functioning of young people with 22q11DS (KileyBrabeck and Sobin 2006).

To conclude, the current study provides an important first step in identifying the social-perceptual and socialcognitive mechanisms associated with social competence in 22q11DS. We found that theory of mind skills are related to parent-rated social competence and emotional problems among children and adolescents with 22q11DS. Whilst people with 22q11DS may have general impairments in face processing, our data suggests that young children with 22q11DS may have a developmental delay in acquiring false-belief and mentalising skills, although this may be related to lower intellectual functioning and/or language ability. Finally, studies of the mechanisms underlying social dysfunction among children with 22q11DS will be useful in order to produce targeted management and remediation of social skills in 22q11DS.

Acknowledgements We would like to thank the VCFS-UK support group and all the families that participated in our research project. We would also like to thank Kim Colyvas (University of Newcastle) and Terry Lewin (Hunter New England Health) for the statistical help provided during the analyses stage of the study. Funding for the current study was received from PPP Healthcare. Furthermore, the first author was supported by a National Health Medical Research Council Australian Training Fellowship (455624) and a Hunter Medical Research Institute post-doctoral fellowship from Port Waratah Coal Services. The funding bodies had no further role in the study design; in the collection, analysis and interpretation of data; in the writing of the report; and in the decision to submit the paper for publication.

\section{References}

Andersson F, Glaser B, Spiridon M, Debbané M, Vuilleumier P, Eliez $\mathrm{S}$. Impaired activation of face processing networks revealed by functional magnetic resonance imaging in 22q11.2 deletion syndrome. Biol Psychiatry. 2008;63(1):49-57.

Baron-Cohen S, Leslie A, Frith U. Does the autistic child have a "theory of mind"? Cognition. 1985;21(1):37-46.

Bassett A, Caluseriu O, Weksberg R, Young D, Chow E. Catechol-omethyl transferase and expression of schizophrenia in 73 adults with 22q11 deletion syndrome. Biol Psychiatry. 2007;61:1135-40.

Bishop DVM. The test for reception of grammar (TROG). 2nd edition. Published by the author and available from Age and Cognitive Performance Research Centre, University of Manchester, M13 9PL, UK; 1983.

Bruce V, Young A. Understanding face recognition. Br J Psychol. 1986;77:305-27.

Bruce V, Campbell RN, Doherty-Sneddon G, Import A, Langton S, McAuley $\mathrm{S}$, et al. Testing face processing skills in children. $\mathrm{Br} \mathrm{J}$ Dev Psychol. 2000;18:319-33.

Campbell L. Brain, behaviour and cognition in 22q11.2 deletion syndrome (22qDS). London: University of London, King's College London; 2006.

Campbell L, Daly E, Toal F, Stevens A, Azuma R, Catani M, et al. Brain and behaviour in children with 22q11.2 deletion syndrome: a volumetric and voxel-based morphometry MRI study. Brain. 2006;129(5):1218-28.

Campbell L, Stevens A, Daly E, Toal F, Azuma R, Karmiloff-Smith A, et al. A comparative study of cognition and brain anatomy between two neurodevelopmental disorders: 22q11.2 deletion syndrome and Williams syndrome. Neuropsychologia. 2009;47 (4):1034-44.

Campbell L, McCabe K, Leadbeater K, Schall U, Loughland C, Rich D. Visual scanning of faces in 22q11.2 deletion syndrome: 
attention to the mouth or the eyes? Psychiatry Res. 2010a;177(12):211-5.

Campbell L, McCabe K, Leadbeater K, Schall U, Loughland C, Rich D. Visual scanning of faces in 22q11.2 deletion syndrome: Attention to the mouth or the eyes? Psychiatry Research. 2010;177(1-2):211-5. doi:10.1016/j.psychres.2009.06.007.

Castelli F, Frith C, Happe F, Frith U. Autism, Asperger syndrome and brain mechanisms for the attribution of mental states to animated shapes. Brain. 2002;125:1839-49.

Chow E, Watson M, Young D, Bassett A. Neurocognitive profile in 22q11 deletion syndrome and schizophrenia. Schizophr Res. 2006;87:270-8.

Dunn LM, Whetton C, Burley J. The British picture vocabulary scale (2nd edition). NFER-Nelson Publishing company Ltd, UK; 1982.

Fine S, Weissman A, Gerdes M, Pinto-Martin J, Zackai E, McDonaldMcGinn D, et al. Autism spectrum disorders and symptoms in children with molecularly confirmed 22q11.2 deletion syndrome. J Autism Dev Disord. 2005;35(4):461-70.

Golding-Kushner K, Weller G, Shprintzen R. Velo-cardio-facial syndrome: language and psychological profiles. J Craniofac Genet Dev Biol. 1985;5(3):259-66.

Goodman R, Ford T, Simmons H, Gatward R, Meltzer H. Using the strengths and difficulties questionnaire (SDQ) to screen for child psychiatric disorders in a community sample. Br J Psychiatry. 2000;177:534-9.

Gopnik A, Astington J. Children's understanding of representational change and its relation to the understanding of false belief and the appearance reality distinction. Child Dev. 1988;59:26-37.

Gothelf D, Presburger G, Levy D, Nahmani A, Burg M, Berant M, et al. Genetic, developmental, and physical factors associated with attention deficit hyperactivity disorder in patients with velocardiofacial syndrome. Am J Med Genet B Neuropsychiatr Genet. 2004;126B(1):116-21

Happé F. Autism, an introduction to psychological theory. London: UCL Press Ltd; 1994.

Haxby J, Hoffman E, Gobbini I. The distributed human neural system for face perception. Trends Cogn Sci. 2000;4:223-33.

Heineman-de Boer J, Van Haelst M, Cordia-de Haan M, Beemer F. Behavior problems and personality aspects of 40 children with velo-cardio-facial syndrome. Genet Couns. 1999;1:1089-93.

Henry J, van Amelsvoort T, Morris R, Owen M, Murphy D, Murphy $\mathrm{K}$. An investigation of the neuropsychological profile in adults with velo-cardio-facial syndrome (VCFS). Neuropsychologia. 2002;40(5):471-8.

Hughes C, Adlam A, Happe F, Jackson J, Taylor A, Caspi A. Good test-retest reliability for standard and advanced false-belief tasks across a wide range of abilities. J Child Psychol Psychiatry. 2000;41:483-90

Iarocci G, Yager J, Elfers T. What gene-environment interactions can tell us about social competence in typical and atypical populations. Brain Cogn. 2007;65:112-27.

Jablensky A. Epidemiology of schizophrenia: the global burden of disease and disability. Eur Arch Psychiatry Clin Neurosci. 2000;250(6):274-85.

Jolliffe T, Baron-Cohen S. The Strange Stories test: a replication with high-functioning adults with autism or Asperger syndrome. J Autism Dev Disord. 1999;29(5):395-406.

Kiley-Brabeck K, Sobin C. Social skills and executive function deficits in children with the 22q11 deletion syndrome. Appl Neuropsychol. 2006;13:258-68.

Lajiness-O'Neill R, Beaulieu I, Titus J, Asamoah A, Bigler E, Bawle E. Memory and learning in children with 22q11.2 deletion syndrome: evidence for ventral and dorsal stream disruption? Child Neuropsychology. 2005;11(1):55-71.

Murphy K. Schizophrenia and velo-cardio-facial syndrome. Lancet. 2002;359(9304):426-30.

Murphy K. Annotation: velo-cardio-facial syndrome. J Child Psychol Psychiatry. 2005;45(6):563-71.

Niklasson L, Rasmussen P, Oskarsdottir S, Gillberg C. Chromosome 22q11 deletion syndrome (CATCH 22): neuropsychiatric and neuropsychological aspects. Dev Med Child Neurol. 2002;44:44 50

Perner J, Wimmer H. John thinks that mary thinks that - attribution of 2nd-order beliefs by 5-Year-Old to 10-Year-Old Children. J Exp Child Psychol. 1985;39(3):437-71.

Posamentier M, Abdi H. Processing faces and facial expressions. Neuropsychol Rev. 2003;13(3):113-43.

Premack D, Woodruff G. Does the chimpanzee have a theory of mind? Behav Brain Sci. 1978;1:515-26.

Sands JR, Harrow M. Vulnerability to psychosis in unipolar major depression: is premorbid functioning involved? Am J Psychiatry. 1995;152(7):1009-15. 1995 July 1.

Shprintzen R. Velo-cardio-facial syndrome: a distinctive behavioural phenotype. Ment Retard Dev Disabil Res Rev. 2000;6(2):142-7.

Shprintzen R. Velo-cardio-facial syndrome. Prog Pediatr Cardiol. 2005;20:187-93.

Siegal M, Varley R. Neural systems involved in "theory of mind". Nature Review Neuroscience. 2002;3(6):463-71.

Swillen A, Devriendt K, Legius E, Eyskens B, Dumoulin M, Gewillig $\mathrm{M}$, et al. Intelligence and psychosocial adjustment in velocardiofacial syndrome: a study of 37 children and adolescents with VCFS. J Med Genet. 1997;34(6):453-8.

Swillen A, Devriendt K, Legius E, Prinzie P, Vogels A, Ghesquiere P. The behavioural phenotype in velo-cardio-facial syndrome (VCFS): from infancy to adolescence. Genet Couns. 1999;10 (1):79-88.

Swillen A, Vogels A, Devriendt K, Fryns JP. Chromosome 22q11 deletion syndrome: update and review of the clinical features, cognitive-behavioral spectrum, and psychiatric complications. Am J Med Genet. 2000;97:128-35.

Tager-Flusberg H, Sullivan K. A componential view of theory of mind: evidence from Williams syndrome. Cognition. 2000;76:59-89.

Tager-Flusberg H, Plesa-Skwerer D, Faja S, Joseph R. People with Williams syndrome process faces holistically. Cognition. 2003;89:11-24.

van Amelsvoort T, Daly E, Robertson D, Suckling J, Ng V, Critchley H, Owen M, Henry J, Murphy KC, Murphy DGM. Structural brain abnormalities associated with deletion at chromosome 22q11: quantitative neuroimaging study of adults with velocardio-facial syndrome. Br J Psych. 2001;178:412-419.

van Amelsvoort T, Schmitz N, Daly E, Deeley Q, Critchley H, Henry $\mathrm{J}$, et al. Processing facial emotions in adults with velo-cardiofacial syndrome: functional magnetic resonance imaging. $\mathrm{Br} \mathrm{J}$ Psychiatry. 2006;189:560-1.

Vorstman J, Morcus M, Duijff S, Klaassen P, Heineman-de Boer J, Beemer F. The 22q11.2 deletion in children: high rate of autistic disorders and early onset of psychotic symptoms. J Am Acad Child Adolesc Psychiatry. 2006;45(9):1104-13.

Wang Y-G, Wang Y-Q, Chen S-L, Zhu C-Y. Theory of mind disability in major depression with or without psychotic symptoms: a componential view. Psychiatry Res. 2008;161 (2):153-61.

Wechsler D. Wechsler Intelligence Scales for Children - third edition. San Antonio, Texas: The Psychological Corporation; 1991. 